\title{
Industrialisierung und Urbanisierung ökologisch modernisieren
}

\author{
Die unaufhaltsam wachsenden Metropolen \\ der Entwicklungsländer stehen vor gravieren- \\ den Problemen der Ver- und Entsorgung. \\ Durch den Aufbau von Kompetenzen bei Ak- \\ teuren aus Wirtschaft und öffentlicher Verwal- \\ tung im Feld der Ressourceneffizienz soll die- \\ ser Entwicklung entgegengewirkt werden. \\ Von Ute Böttcher
}

\begin{abstract}
n den Städten der Entwicklungs- und Schwellenländer sind neben den Haushalten und Stadtverwaltungen die Industrieunternehmen wichtige Akteure. Sie zeichnen sich durch Energieerzeugung mit hohen Emissionsgraden und eine große Gefährdung der Umwelt durch Verschmutzungen aus.

Sowohl im Hinblick auf den Ressourcenverbrauch, als auch im Hinblick auf Sicherheitsaspekte ist eine ökologisch effiziente Industrieproduktion und eine (Entsorgungs- und Versorgungs-) Infrastruktur in Städten wichtig. Umso mehr, da die ökologischen Auswirkungen großer Ballungsräume sich nicht nur auf die urbanen Gebiete und ihre Bevölkerung beschränken, sondern auch im Umland wirksam werden. Insbesondere Prozesse, die im Zusammenhang mit dem Verbrauch von natürlichen Ressourcen stehen, wirken sich negativ auf die ländlichen Regionen aus.
\end{abstract}

\section{Globale Verantwortung für Ressourceneffizienz}

Auf dem Weltgipfel Johannesburg 2002 verabschiedeten die Staatsoberhäupter ein Programm zur Implementierung nachhaltiger Konsum- und Produktionsmuster. Dieses wurde auf der Startkonferenz in Marrakesch 2003 mit konkreten Zielen wie der Vernetzung der Akteure sowie dem Austausch von Informationen und Wissen verknüpft. Alle Staaten wurden aufgefordert, auf nationaler wie internationaler Ebene tätig zu werden. 2011 soll ein Zehn-Jahresprogramm durch den sogenannten Marrakesch-Prozess erarbeitet und verabschiedet sein.

Auch der Rat der OECD appellierte an seine Mitgliedsstaaten, Schritte zu einer verbesserten Information über Materialflüsse zu unternehmen, um die Effizienz der eingesetzten Ressourcen zu fördern. Die 3-R Initiative der G8 hat ebenfalls die effiziente Nutzung von Ressourcen und Materialien zum Ziel. Die Vermeidung, Verwendung und Verwertung (3R-Redu- ce, Reuse, Recycle) von Stoffen soll dabei gefördert werden. Die verstärkte Kooperation von Industrie- und Entwicklungsländern wird dabei von allen Beteiligten gefordert.

Die verschiedenen technischen Ansätze und Verfahren für eine Verminderung des Ressourcenverbrauchs existieren bereits seit Jahren. Themen wie Cleaner Production (CP) und Stoffstrommanagement bilden oftmals Bestandteile des industriellen Umweltmanagements. Als Alternative zur vorherrschenden Fokussierung auf sogenannte „End-of-pipe-Technologien“, wie zum Beispiel industrielle oder städtische Kläranlagen, welche die Umweltauswirkungen durch nachgeschaltete Maßnahmen zu vermindern suchen, gewinnt das Konzept der Ressourceneffizienz an Attraktivität. Gleichzeitig können die Qualität der produzierten Güter erhöht, mit der Produktion verbundene Risiken minimiert und Recyclingpotenziale erschlossen werden. Dabei soll erreicht werden, den Ressourcenverbrauch vom Wirtschaftswachstum zu entkoppeln. Um die Umweltverträglichkeit eines Produkts oder einer Dienstleistung zu ermitteln, ist eine Betrachtung der gesamten Wertschöpfungskette notwendig. Ergebnis ist eine Dematerialisierung des Produktionsprozesses, d.h. pro Produktionseinheit werden weniger Energie und Rohstoffe eingesetzt.

Allerdings kommt es nun weltweit darauf an, das Potenzial dieser technischen Lösungen und Verfahren mit den Aufgaben von Industrieunternehmen und Regierungen so zu verknüpfen, dass Ressourceneffizienz für diese Akteure ein erklärtes Ziel darstellt. Ressourceneffizienz als Konzept umzusetzen benötigt Menschen, die diese Technik und Verfahren anwenden können und die im Rahmen von Aushandlungsprozessen Effizienzpotenziale erkennen und nutzen. Diese Effizienzpotenziale sind bislang kaum ausgeschöpft und können um ein Vielfaches höher sein als das, was durch weitere Verbesserungen der Verfahren und technische Entwicklungen zu erreichen ist.

\section{Partnerschaft und Kooperation für Ressourceneffizienz}

Für die Entwicklungszusammenarbeit, insbesondere die Technische Zusammenarbeit, kommt der Vernetzung der Akteure und dem Transfer von Wissen und Technologien zur Umsetzung politischer Ziele, wie das der Ressourceneffizienz, eine besondere Bedeutung zu. Sie verbindet Industrie- und Entwicklungsländer, indem sie Kompetenzen schafft, Fähigkeiten vermittelt und den Transfer von Technologien organisiert. Dem Kapazitätenaufbau kommt dabei die wichtigste Rolle zu. In verschiedenen Projekten der technischen Zusammenarbeit $\rightarrow$ 
hat das Thema Ressourceneffizienz in den letzten Jahren eine große Bedeutung erlangt.

Stadtverwaltungen werden dabei unterstützt, durch die Einführung eines kommunalen Umweltmanagements ihre Abfallströme zu organisieren, und in kleinen oder mittelständischen Unternehmen werden mithilfe des industriellen Umweltmanagements und der Anwendung von Methoden des Profitablen Umweltmanagements oder Ökoprofits Einsparungen durch eine effizientere Nutzung der Ressourcen erzielt. Auch ganze Industrieparks werden bei ihrer ökologischen Modernisierung unterstützt.

Die Erfolge hier waren schon beträchtlich. Dennoch gilt, dass in der Vergangenheit durch die oftmals getrennte Beratung von öffentlichen Trägern und Industrieunternehmen in Fragen des Umweltmanagements und nachhaltigen Wirtschaftens Effizienzpotenziale nicht genutzt wurden, die bei einer gemeinsamen Betrachtung möglich gewesen wären. Deshalb basiert das aktuelle Konzept auf einer Vernetzung der Akteure mit dem Ziel einer gesteigerten Ressourceneffizienz durch Nutzung von Synergien. Das klingt zunächst einfach, ist es aber nicht. Denn neben der Vermittlung von technischem Wissen und der Vorstellung von technischen Lösungen bei den öffentlichen und privaten Partnern in den Entwicklungsländern geht es nun darum, diese bislang eher getrennt agierenden Akteure zusammenzubringen und Methoden für sie bereitzustellen, wie sie durch Aushandlungsprozesse an einer gemeinsamen Umsetzung von Ressourceneffizienz arbeiten können.

Abbildung 1: Ressourceneffizienz durch Kapazitätenaufbau

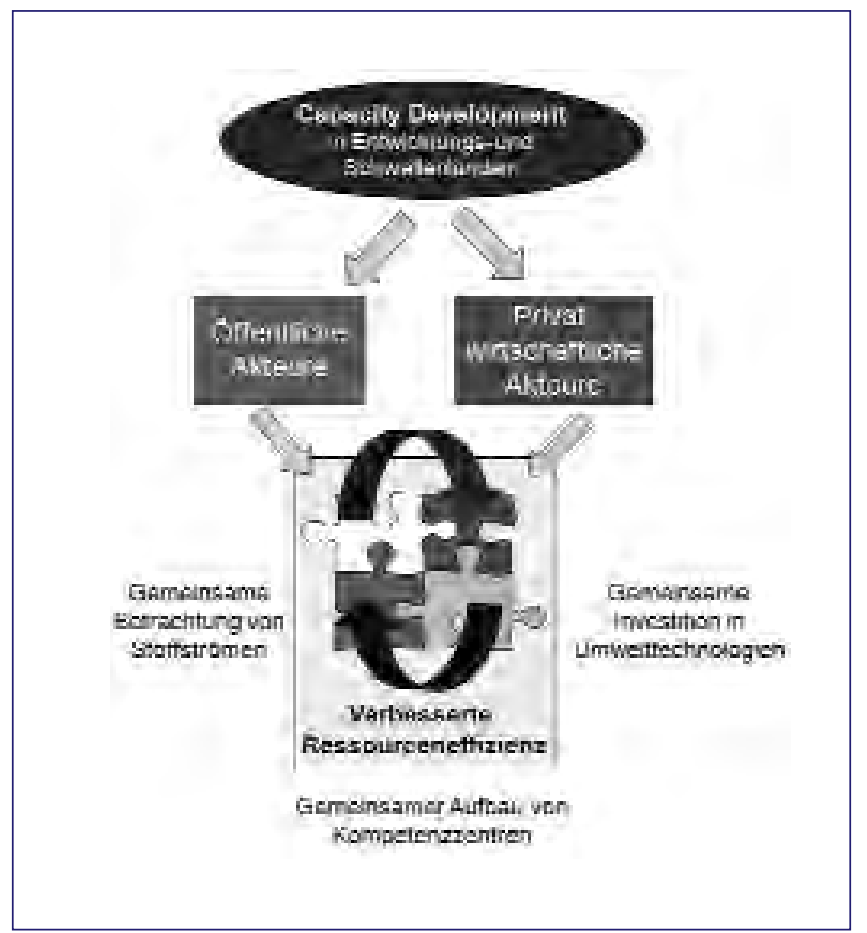

Quelle: Eigene Darstellung

\section{Betrachtung von Stoffströmen}

Die Initiierung von Partnerschaften zwischen privaten und öffentlichen Akteuren birgt viele Potenziale. Während bislang zum Beispiel die Stoffströme einer Stadt und eines angrenzenden Industriegebietes separat betrachtet wurden, ist die gemeinsame Analyse ein erster Schritt, um zukünftige Maßnahmen in Kooperation zu planen. So bleibt es nicht bei einer Bereitstellung von Land und Infrastruktur seitens der Kommune oder aber einer separaten Kreislaufwirtschaft in einem Industriegebiet. Vielmehr werden vorhandene Ansätze auf beiden Seiten vernetzt und mögliche Synergiepotenziale aufgedeckt. Denkbare Kooperationsbereiche umfassen beispielsweise die Wasserund Abfallwirtschaft, die Beseitigung von Altlasten, die Energieversorgung und das städtische Transportwesen. Kostensenkungen und höhere Effektivität können durch eine Steigerung der Ressourceneffizienz auf beiden Seiten erreicht werden. Im Rahmen integrierter Projekte werden Ansätze der Wirtschaftsförderung, der regionalen Entwicklung und des städtisch-industriellen Umweltmanagements zusammengeführt. Die Implementierung ressourceneffizienter Verfahren und Prozesse führt dabei neben einer Reduzierung der Umweltauswirkungen auch zu weiteren Kostensenkungen und stärkt somit die Wettbewerbsfähigkeit der Betriebe und Institutionen. Insbesondere kleine und mittlere Unternehmen werden so in ihrer marktwirtschaftlichen Position gestärkt.

Voraussetzung für eine erfolgreiche Kooperation öffentlicher und privater Akteure ist das Vorhandensein von Kompetenzen und Fähigkeiten auf beiden Seiten. Eine nachhaltige Entwicklung wird erst möglich, wenn Aktivitäten und Strategien effizient koordiniert und gesteuert werden. Insbesondere im Bereich Ressourceneffizienz und umweltschonender Produktionsprozesse bedarf es spezieller Kenntnisse, die in Entwicklungsländern oftmals nicht im erforderlichen Ausmaß vorhanden sind. Deshalb muss dem Kapazitätenaufbau, im Englischen „Capacity Development“, die besondere Aufmerksamkeit gelten. Ziel des „Capacity Development“ ist es, die Handlungskompetenz der involvierten Akteure zu stärken und Fähigkeiten von Menschen und Organisationen auszubauen. Langfristig wird so die Fähigkeit zur Selbsthilfe eines Landes gestärkt.

\section{Investition in Umwelttechnologien}

Im Zusammenhang mit dem komplexen Thema Ressourceneffizienz bedeutet dies neben der gemeinsamen Betrachtung von Stoffströmen und deren Analyse, dass sich Vertreter der Wirtschaft und der lokalen Verwaltung darauf einigen, bestimmte Umwelttechnologien, sei es eine Kläranlage oder ein Recyclinghof, gemeinsam anzuschaffen und zu nutzen. So wird es auch nicht ausbleiben, dass Kooperation und Partnerschaft durch neue innovative Gesetze, Anreize oder freiwillige Vereinbarungen flankiert werden. Um öffentliche und private Akteure zu gemeinsamen Handlungen, zum Beispiel einer gemeinsamen Investitionsentscheidung in den Entwicklungsländern 
zu befähigen, muss ihr Wissen über Umwelttechnologien erweitert werden und sie müssen in die Lage versetzt werden, selbst zu entscheiden, welche Umwelttechnologie für sie angemessen und hilfreich ist. Darüber hinaus sind Finanzierungskonzepte gefragt, die es den öffentlichen und privaten Akteuren ermöglichen, gemeinsame Entscheidungen zur Anwendung von Umwelttechnologien zu treffen und gemeinsame Finanzierungsmodelle zu erarbeiten.

\section{Aufbau von Kompetenzzentren}

Ein Ansatz zur Wissensverbreitung über ressourceneffizientes Wirtschaften kann zum Beispiel die Einrichtung von Kompetenzzentren in den Partnerländern sein. Spezifische Schulungen für Personal aus Kommunen und der Privatwirtschaft, nicht nur bezogen auf die technischen Lösungen für mehr Ressourceneffizienz, sondern auch für den Aufbau von Netzwerken, Unternehmergruppen zur hier angesprochenen Stärkung der gemeinsamen Handlungskompetenz könnten dazu beitragen, die Industrialisierung und Urbanisierung dadurch ressourceneffizient zu gestalten.

Bereits existierende Kompetenzzentren aus der Abfallwirtschaft oder aus dem Bereich „Cleaner Production“ müssten sukzessive Konzepte und Modelle für öffentliche und private Akteure gemeinsam anbieten. Des Weiteren sind Methoden zu entwickeln, wie Stoffströme zusammengeführt werden können, welche Effizienzpotenziale bei einer gemeinsamen Betrachtung bestehen und welche Finanzierungsmodelle es für gemeinsame Investitionsentscheidungen gibt. Dies gilt auch für alle bestehenden und noch zu gründenden überregionalen Kompetenzzentren.

Die Vorteile einer ressourceneffizienten Konsum- und Produktionsweise erscheinen uns vielfältig. Ein verminderter Ressourcenverbrauch schont die natürlichen Vorkommen und belastet die Umweltmedien in geringerem Maße durch Emissionen und Stoffaus- bzw. -einträge. Insbesondere in Entwicklungsländern, in denen oftmals große Teile der Bevölkerung aufgrund von Armut in risikoexponierten Lagen leben, kann die Lebensqualität gesteigert werden. Daneben ergeben sich konkrete wirtschaftliche Vorteile. Da Rohstoffe und andere Materialien einen nicht unwesentlichen Teil der Produktionskosten darstellen, können die Kosten bei einer effizienten Nutzung reduziert werden. Dadurch entstehen Wettbewerbsvorteile gegenüber herkömmlich agierenden Unternehmen. Im Fall von privaten und öffentlichen Partnerschaften können darüber hinaus Investitions- und Betriebskosten geteilt werden.

Unternehmen und Institutionen, die ressourceneffizient wirtschaften, haben nicht zuletzt die Möglichkeit, ihr „Corporate Image“ zu verbessern und dadurch ihre Kundenattraktivität zu erhöhen. Methodisch wird es dafür notwendig sein, bestehende Ansätze des städtischen und des industriellen Umweltmanagements zusammenzuführen und neue Modelle zu entwickeln, wie öffentliche und private Akteure die brachliegenden Effizienzpotenziale erkennen können und wie Ressourceneffi- zienz als gemeinsame Handlungsoption umzusetzen ist. Zukünftig sind Konzepte gefragt, die in die methodische Vorgehensweise bei der Verknüpfung der Akteure für eine Ressourceneffizienzsteigerung sowie in die Erfolgsbewertung von Ressourceneffizienz investieren.

Um die Wirkungen ressourceneffizienter Produktion festzustellen, sind zuverlässige Messmethoden und -konzepte notwendig. Einen Ansatz stellt das MIPS-Konzept (Materialinput pro Serviceeinheit) dar. Es erfasst den Naturverbrauch eines Produktes oder einer Dienstleistung entlang der gesamten Wertschöpfungskette und ermittelt so den Grad der Umweltbeeinträchtigung. Ein anderes Konzept ist der „ökologische Fußabdruck“, welcher ebenfalls dem Gesamtrechnungsansatz zugerechnet werden kann. Er misst das Verhältnis von globaler Biokapazität (Fläche x Bioproduktivität) und dem Ressourcenverbrauch sowie dessen Intensität. Diesem Ansatz liegt die Annahme zugrunde, dass Nachhaltigkeit nur dann gewährleistet ist, wenn der Verbrauch natürlicher Ressourcen die regenerative Kapazität der Erde nicht übersteigt. Methoden der Wirkungsmessung werden an Bedeutung gewinnen, um die Ressourceneffizienzsteigerungen in räumlich definierten Gebieten zu beschreiben.

\section{Industrialisierung ressourceneffizient gestalten}

Ein Risiko, welches mit der Einführung von ressourceneffizienten Technologien verbunden ist, stellt der Reboundeffekt dar. Ein Beispiel sind Hybridfahrzeuge, die aufgrund ihres geringen Treibstoffverbrauchs auf zusätzlichen Strecken eingesetzt werden könnten - die vormals erzielten Einsparungen werden hier durch vermehrten Konsum bzw. Nutzung kompensiert, Umweltbelastungen werden nicht reduziert, sondern eventuell sogar erhöht. Ein weiteres Beispiel stellt die Nachfrage nach Computern dar: Sie werden in immer effizienteren Herstellungsprozessen mit sinkenden Preisen auf dem Markt angeboten, sodass mehr Menschen in der Lage sind, sich einen Computer zu leisten. Die gestiegene Nachfrage kann hier die Einsparungen des Produktionsprozesses (über-)kompensieren. So wird durch die verstärkte Nutzung eventuell ein höherer Anteil an Ressourcen verbraucht, als es in einem Fall ohne ressourceneffizientes Wirtschaften der Fall wäre. Dem kann nur durch eine Bewusstseinsschaffung und Änderung des Konsumverhaltens begegnet werden. Um künftig bei einer Umsetzung des Ressourceneffizienzansatzes in den Entwicklungs- und Schwellenländern den Reboundeffekt möglichst auszuschließen, besteht noch weiterer Forschungsbedarf.

Im Rahmen des G8-Gipfels in Heiligendamm im Juni 2007 hat China erklärt, dass die Hauptverantwortung für den durch Umweltbelastungen getriebenen Klimawandel bei den reichen Industrienationen liege. Schwellenländer können sich nicht einschränkenden Vereinbarungen (wie zum Beispiel die Begrenzung des $\mathrm{CO}_{2}$-Ausstoßes) unterwerfen, da diese ihr Wirtschaftswachstum (also ihre Aufholjagd) behindern würden. Wenn wir, als industrialisierte Länder, eine Verantwortung haben, dann $\rightarrow$ 
sicherlich diejenige, Schwellen- und Entwicklungsländer bei einer effizienteren Nutzung der Ressourcen zu unterstützen, eben durch eine technologisch machbare Entkopplung von Wirtschaftswachstum und Ressourcenverbrauch sowie durch die Nutzung von Effizienzpotenzialen durch Partnerschaften und Kooperationen auf allen Ebenen und zwischen allen Beteiligten.

Nur so kann der weltweite Ressourcenverbrauch dauerhaft gesenkt und Industrialisierung und Urbanisierung ökologisch modernisiert werden.

\section{Literatur}

Bundesministerium für Umwelt, Naturschutz und Reaktorsicherheit (BMU) / IG Metall / Wuppertal Institut für Klima, Umwelt, Energie (Hrsg.): Ressourceneffizienz - Innovation für Umwelt und Arbeit. Wuppertal 2006. (http://www.wupperinst.org/uploads/tx_wibeitrag/ressourceneff_brosch.pdf)

Deutsche Gesellschaft für Technische Zusammenarbeit (GTZ) (Hrsg.): Policy Instruments for Resource Efficiency. Towards Sustainable Consumption and Production. Eschborn 2006. (http://www.gtz.de/de/dokumente/eneco-efficiency-policy-instruments.pdf)

Deutsche Gesellschaft für Technische Zusammenarbeit (GTZ) (Hrsg.): Schlussbericht zur Durchführung des Vorhabens „Entwicklung öko-industrieller Parks und Netzwerke“. Eschborn 2004.

Earthscan / UNDP (Hrsg.): Ownership, Leadership and Transformation. Can we do better for capacity development? Paris 2003. (http://www.capacity.undp.org/indexAction.cfm?module=Library\&action=GetFile\&DocumentAttachmentID=1001)

Effizienz-Agentur NRW (Hrsg.): Ressourceneffizienz. Strategie für umweltgerechtes Wirtschaften. Düsseldorf 2006. (http://www.efanrw.de/downloads/publikationen/Weissbuch_final.pdf)

International Bank for Reconstruction and Development / World Bank (Hrsg.): Global Economic Prospects. Realizing the Development Promise of the Doha Agenda. Washington 2003.

(http://siteresources.worldbank.org/INTRGEP2004/Resources/gep2004fu Iltext.pdf)

Lowe, E. A.: Eco-industrial Park Handbook for Asian Developing Countries. Report to Asian Development Bank. Oakland 2001.

OECD (Hrsg.): Recommendation of the Council on Material Flows and Resource Productivity. Paris 2004. (http://www.oecd.org/dataoecd/3/63/31571298.pdf)
Ritthoff, M. / Rohn, H. / Liedtke, C.: MIPS berechnen. Ressourcenproduktivität von Produkten und Dienstleistungen. Wuppertal 2002. (http://www.wupperinst.org/uploads/tx_wibeitrag/ws27.pdf)

Sustainable Europe Research Institute (SERI): Ressourceneffizienz und Nachhaltigkeit. Thema des Monats 5/2006 des Portals Nachhaltigkeit.at. (http://www.nachhaltigkeit.at/reportagen.php3?id=6)

Sustainable Europe Research Institute (SERI): Überblick über Dematerialisierung, Ökoeffizienz und Zero Emission. Thema des Monats 05/2001 des Portals Nachhaltigkeit.at.

(http://www.nachhaltigkeit.at/bibliothek/pdf/monatsthemen2001/mainM 01_05.pdf)

UNEP (Hrsg.): Sustainable Consumption \& Production - How Development Agencies make a difference. Review of Development Agencies and SCP-related projects. Paris 2006.

Vorholz, F.: Sind die Menschen noch zu retten? DIE ZEIT, 47/2006. (http://www.zeit.de/2006/47/Klimakonferenz)

Weizsäcker, E. U. von / Lovins, A. / Lovins, H.: Faktor Vier. Doppelter Wohlstand - halbierter Naturverbrauch. München 1995.

WWF International / Zoological Society of London / Global Footprint Network (Hrsg.): Living Planet Report 2006. Gland, London, Oakland 2006. (http://assets.panda.org/downloads/living_planet_report.pdf)

\section{AUTORIN + KONTAKT}

Dr. Ute Böttcher ist Seniorfachplanerin in der Abteilung Umwelt und Infrastruktur der Gesellschaft für Technische Zusammenarbeit (GTZ) und verantwortlich für die Entwicklung und Evaluierung von Projekten und Programmen des städtischen und industriellen Umweltmanagements.

Ute Böttcher, Deutsche Gesellschaft für Technische Zusammenarbeit (GTZ) $\mathrm{GmbH}$, Dag-Hammarskjöld-Weg 1-5, 65760 Eschborn, Tel.: +496196 791200, E-Mail: ute.boettcher@gtz.de

\section{politische ökologie}

Die Zeitschrift für Querdenker und Vordenkerinnen

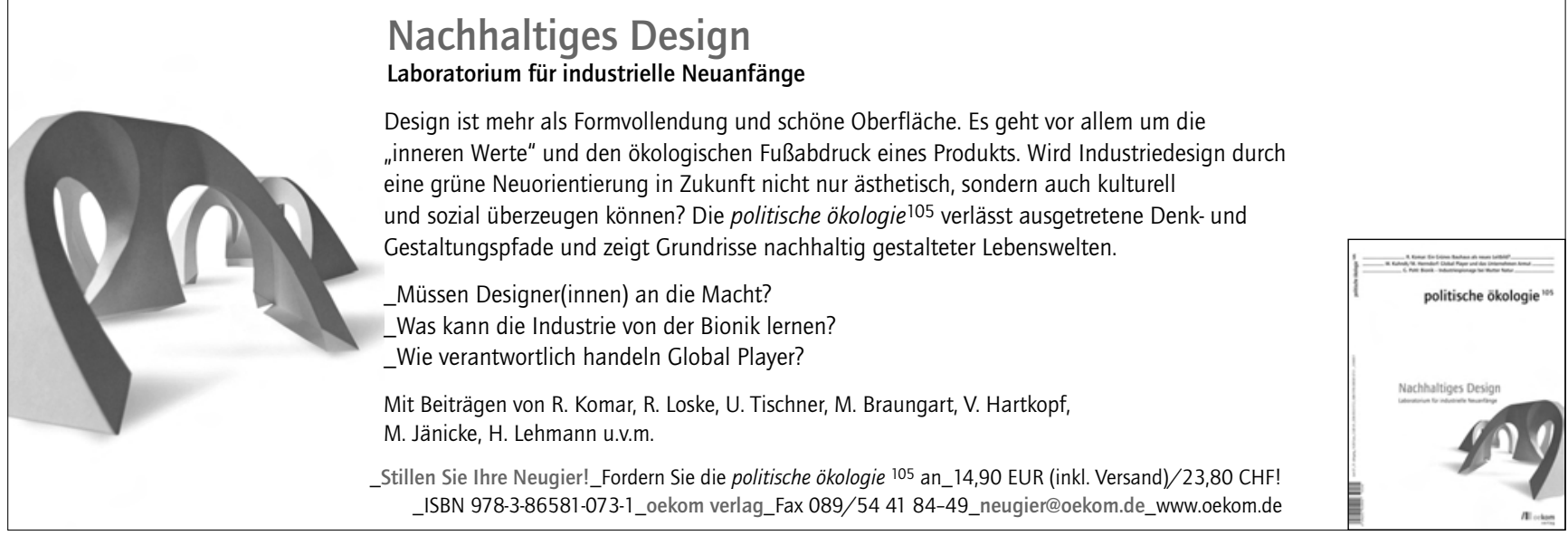


(c) 20I0 Authors; licensee IÖW and oekom verlag. This is an article distributed under the terms of the Creative Commons Attribution Non-Commercial No Derivates License (http://creativecommons.org/licenses/by-nc-nd/3.o/), which permits unrestricted use, distribution, and reproduction in any medium, provided the original work is properly cited. 